\title{
Discrimination of relative numerosity by pigeons
}

\author{
W. K. HONIG and KAREN E. STEWART \\ Dalhousie University, Halifax, Nova Scotia, Canada
}

\begin{abstract}
In three experiments, pigeons were trained to discriminate between uniform arrays of two elements that differed in color, form, or size. They were then tested with arrays that contained different proportions of the two elements on these dimensions. In all cases, orderly discrimination gradients reflected these proportions. The discrimination readily transferred to new arrays with similar stimuli, but with different total numbers of elements. In Experiment 4, the pigeons were taught to discriminate between two groups of categorical stimuli: pictures of birds and pictures of flowers. A test with different proportions of each again produced a gradient based on relative numerosity. Experiment 5 demonstrated transfer of stimulus control on the numerosity dimension when pigeons were trained with one set of instances from two categories, and then were tested with new instances from the same categories.
\end{abstract}

In a thoughtful and thorough review of research on counting in animals, Davis and Memmott (1982) distinguished between number discrimination and counting. Counting involves a precise tagging of each item in a display or a sequence of events, and a partitioning of items to distinguish between those already counted and those that remain to be counted. Convincing evidence for these processes in animals is sparse, and even when it can be found, the number of items or events that animals can count reliably does not extend much beyond three or four. Most species do not seem to possess the symbolic capacity required to enumerate the individual items in an array. Number discrimination, on the other hand, does not involve the rigorous criteria of true counting: this process

refers to an organism's ability to make broad relative judgments such as many versus few. Such judgments are occasionally referred to as "numerosity judgments" or "magnitude estimations." The important point is that number discrimination is an essentially imprecise ability that is not as advanced as counting. (Davis \& Memmott, 1982, pp. 548-549)

Although this is a useful and important distinction, Davis and Memmott did not further discuss the topic of number discrimination. They devoted the rest of their review to counting. This suggests that there has been little research on the discrimination of numerosity, as distinguished from counting.

The imprecise nature of numerosity discriminations (as we will call them) may discourage psychologists from analyzing them experimentally. However, the apparent numerosity of objects in a spatial array is no doubt important for many animals and birds. It is useful to be able

This research was supported by Grant AO-102 from the Natural Sciences and Engineering Research Council of Canada. We thank Heather Schellink for experimental assistance and William Hayes for providing the computer programs. Address correspondence to $\mathrm{W}$. $\mathrm{K}$. Honig, Department of Psychology, Dalhousie University, Halifax, Nova Scotia B3H 4J1, Canada. to discriminate the size of a group or a flock of other animals, the density of trees in a particular area, or the relative numbers of ripe and unripe berries on a branch. As shown by recent research on concept attainment, pigeons readily classify items within several different categories (Edwards \& Honig, 1987; Greene, 1983; Herrnstein, 1979; Herrnstein \& deVilliers, 1980; Herrnstein \& Loveland, 1964; Herrnstein, Loveland, \& Cable, 1976; Lubow, 1974; Siegel \& Honig, 1970; Vaughan \& Herrnstein, 1987). This capacity would be particularly useful for an animal if it could also estimate the quantity, or numerosity, of the items in an array that belong to the category. The present research involved discriminations of this nature. Simple visual stimuli were used in much of the research to facilitate the analysis of this process, but Experiments 4 and 5 involved more complex stimuli from two different categories.

For the proper study of numerosity discriminations, it is important to control confounded variables. If objects are of a constant size, then the exent of the array will be correlated with the number of objects. If this is controlled by adjusting the sizes of individual objects or the average distances between them, then these cues will in turn be confounded with the number of objects. Similar problems would arise with events extended in time, rather than objects extended in space. To obviate these problems, we presented pigeons with arrays of a constant total number of stimuli. These differed in a particular characteristic, namely color, form, or size, or in the more complex features defining categorical stimuli. The relative numerosity of the two classes of stimuli varied among the different arrays, and this provided the dimension for the control of behavior.

\section{GENERAL METHOD}

\author{
Subjects \\ White Carneaux pigeons served as subjects. They were main- \\ tained at about $80 \%$ of their free-feeding weights by daily feeding, \\ but had water freely available. Most of them had served in other
}


experiments involving classical conditioning, or other procedures that had little in common with the present research.

\section{Apparatus}

Two pigeon chambers were used in a sound-attenuated environment. Each contained a response panel that was about $5.1 \mathrm{~cm}$ square, onto which the stimuli were rear-projected. Mixed grain reinforcement was presented for $4 \mathrm{sec}$ in a standard food hopper. Kodak ektagraphic carousel projectors were used to project the stimuli. The procedures were controlled by an Apple Ile computer running on a Pascal program designed by William Hayes.

\section{Stimuli}

The pigeons were presented with square matrices of stimulus elements. These largely filled the response panel. Except for the pictorial stimuli used in Experiments 4 and 5, the matrices could be presented in four different orientations and their mirror images. These orientations, and the stimulus orders, were changed every few sessions in the course of training. When a matrix comprised elements from two different classes, these elements were randomly assigned to the specific locations in the matrix.

\section{Training and Test Procedures}

Each training or test session consisted of a series of trials lasting at least $20 \mathrm{sec}$. The intertrial interval was $10 \mathrm{sec}$. During a trial, a single array was presented. On positive trials, the display ended with the first response after $20 \mathrm{sec}$, which provided the pigeon with $4 \mathrm{sec}$ of access to the grain hopper. Only the responses occurring during the first $20 \mathrm{sec}$ of the trial were counted. Negative trials in discrimination training, and all test trials in a gradient test, ended after $20 \mathrm{sec}$ without reinforcement.

Discrimination training generally involved two uniform arrays of positive and negative elements. In such cases, a session comprised 32 trials, and each array appeared four times in each block of 8 trials. An array was not presented more than three times in a row. In the test sessions that yielded numerosity gradients, the arrays, including the uniform training arrays, comprised several different proportions of the training elements. These proportions are called numerosity values or test values. Each test session was subdivided into four blocks of trials, and each block contained one instance of each numerosity value. Different numbers of test values were presented in different experiments, and are described accordingly. Reinforcement was withheld during test sessions.

\section{Data}

Individual subjects differed in their "typical" rates of responding, and the data were therefore normalized for each subject before they were averaged. When data were obtained from discrimination training with two values, the discrimination ratio represents the proportion of total responses emitted to the positive stimulus. When data were obtained from several values on the dimension of numerosity in testing, the number of responses emitted to the uniform, positive array was assigned a reference value of 1.0 , and all other response totals were calculated and expressed as proportions of that value. In some procedures, two consecutive test sessions were run without reinforcement, and the responses were summed across the sessions before the data were normalized.

\section{EXPERIMENT 1: COLOR DISCRIMINATION}

Experiment 1 employed items of different colors. Color differences are very salient for pigeons, and many experiments have been based on this dimension since the study by Guttman and Kalish (1956).

\section{Method}

Four pigeons were initially used for this experiment. Three of them had little difficulty with the discrimination, and contributed to the present results. One bird reached a high level of discrimination from time to time, but for unknown reasons its performance deteriorated sporadically. Its data are therefore not included.

In the standard arrays, 36 elements were presented. Uniform arrays of red and blue dots were used in training. Projected onto the response panel, the dots had a diameter of $4 \mathrm{~mm}$ and were spaced $11 \mathrm{~mm}$ apart, center-to-center. The test arrays comprised the following proportions of stimuli: 36 red, 0 blue; 30 red, 6 blue; 25 red, 11 blue; 21 red, 15 blue; 15 red, 21 blue; 11 red, 25 blue;

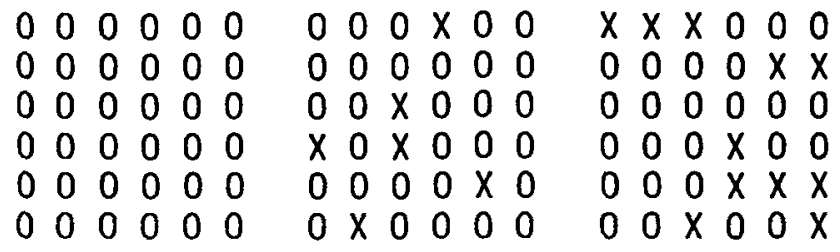

$\times 0 \times 0 \times 0$

$\begin{array}{llllll}0 & 0 & 0 & \times & 0 & 0\end{array}$

$\times 0000 \times 0$

$\times 0 \times 0 \times 0$

$0 \times 0 \times 0 \times 0$

$0 \times \times \times \times 0$

$00 \times \times \times \times$

$x \times x \times 0 \times x$

$x \quad 0 \quad x \quad 0 \quad 0 \quad 0$

$\times \times \times \times \times \times$

$x \times x \quad x \quad 0 \quad x \quad x$

$x \times 00 x$

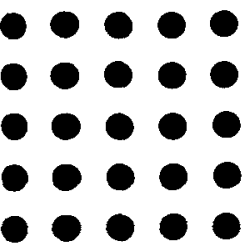

$\begin{array}{llllll}x & x & 0 & 0 & 0 & x\end{array}$

$0 \times \times \times 00$

$00 \times \times \times 0$

$x \times \times \times 0 \times$

$\begin{array}{llllll}0 & \times & 0 & 0 & \times & 0\end{array}$

$\times 000 \times 0$

$x \times 000 \times 0$

$00 \times \times \times \times \times$

$0 \times 0 \times \times 0$

$0 \times \times \times 0 \times$

$\begin{array}{llllll}0 & 0 & 0 & 0 & x & 0\end{array}$

$x \times \times \times \times x$

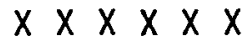

$\begin{array}{llllll}x & x & \times & \times & x & x\end{array}$

$\begin{array}{lllllll}x & x & x & x & x & x\end{array}$

$x \times x \quad x \quad x \quad x \quad x$

$x \times x \times x \times x$

$x \times \times \times \times x$ $x \times x \quad x \quad x \quad x \quad x$

$x 0 \times \times \times x$ 
6 red, 30 blue; 0 red, 36 blue. As indicated above, the dots of different colors were randomly located within each of several arrays for each proportion. One set of such arrays is shown in the upper panel of Figure 1, with Xs and Os representing the different elements. Patterns containing 18 dots of each color were not used at first, but were added in later phases of the research.

The 3 birds from this group were each given at least four gradient tests, interspersed with continued training on the discrimination between the all-red and all-blue arrays. They were tested on the dimension of numerosity only when they attained a high level of discrimination, typically giving $95 \%-99 \%$ of their responses during a session to $\mathrm{S}+$. Seven or eight training sessions were normally carried out between test sessions, but sometimes there were fewer, or even more, depending on the level at which the discrimination was maintained.

\section{Results}

The mean normalized gradients obtained from the four gradient tests given to 3 subjects are shown in Figure 2. The gradients reflect the high level of the discrimination between the uniform arrays, and responding to the test numerosities is correlated with the relative numbers of positive and negative elements. The gradients became somewhat steeper in the course of repeated testing; this is commonly obtained in operant studies of stimulus control. The normalized gradients have been combined into one overall gradient, which shows a rather linear proportional decline in responding between $S+$ and $S-$ on the dimension of numerosity. A combined numerosity gradient based on the mean total responses obtained from each test is presented at the right. This corresponds closely to the mean normalized gradient at the left. Relative numerosity of the elements of different colors seems to control behavior in the manner of a typical simple stimulus dimension, such spectral value (Guttman \& Kalish, 1956), line orientation (Honig, Boneau, Burstein, \& Pennypacker, 1961), or auditory frequency (Jenkins \& Harrison, 1960).

\section{EXPERIMENT 1A: TRANSFER TO ARRAYS OF 64 DOTS}

To determine whether the stimulus control that we had obtained on the dimension of numerosity with 36-dot arrays was based on the actual numbers of positive and negative elements or on their relative proportions, transfer tests were carried out with a larger set of elements, namely, matrices of 64 dots. In randomized patterns of 64 dots, red and blue appeared in the same proportions as they did in the 36-dot arrays. These square patterns, when projected onto the pecking screen, were $59 \mathrm{~mm}$ on each side, and thus covered a slightly larger area than the patterns of 36 dots. Thus, the individual dots were somewhat smaller ( $3 \mathrm{~mm}$ in diameter) and more closely spaced within the matrix ( $8 \mathrm{~mm}$ center-to-center).

After several sessions of further training with the allred and all-blue 36-dot arrays, the pigeons were tested during two successive sessions with the 64-dot arrays. This was the first occasion on which they saw these arrays. A second test of this kind was run with 2 of the birds after six further training sessions with the 36-dot arrays. (One pigeon had fallen behind the other 2 subjects because of a disruption of stimulus control, and was given only one transfer test.)

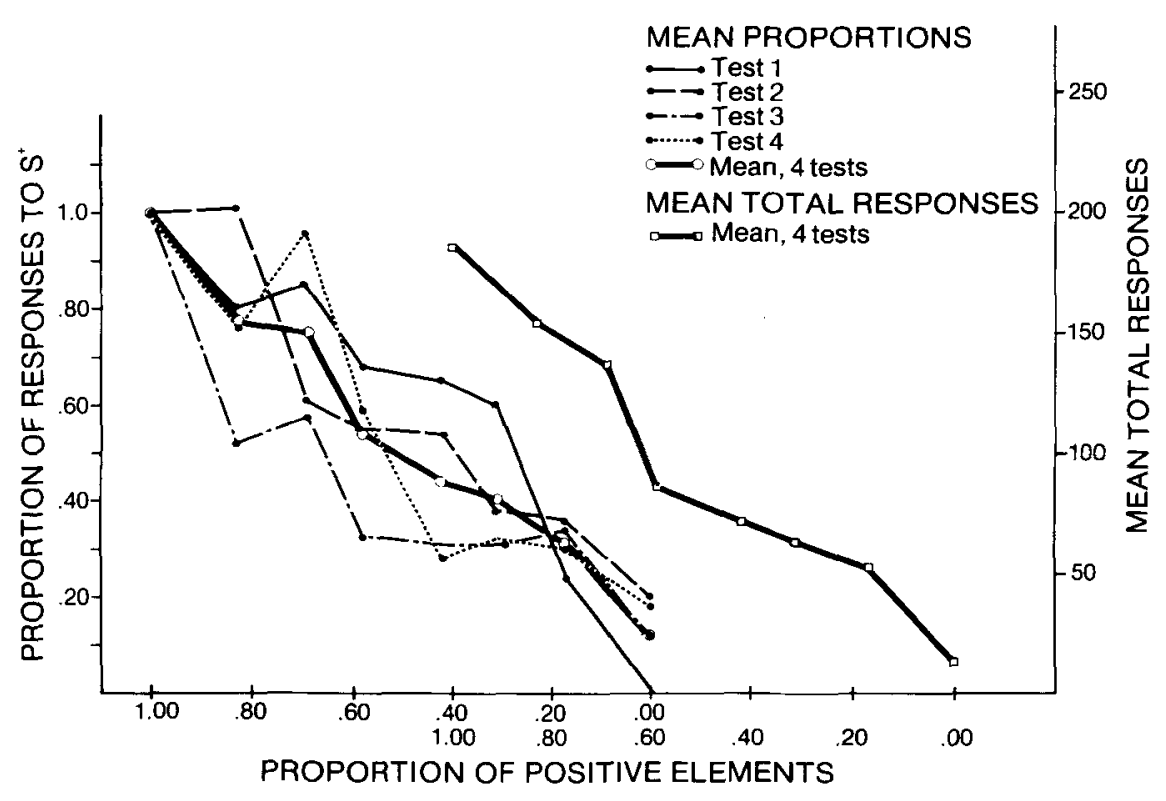

Figure 2. Mean numerosity gradients obtained from 3 pigeons in Experiment 1 with colored dots. The four dashed lines provide results from successive tests. The single solid line is the mean of these gradients. At the right is the corresponding mean gradient of total responses per test. 
Results

Prior to the transfer tests, the discrimination between the training arrays was essentially perfect. The transfer gradients, each based on the total responses from two test sessions, are shown in Figure 3, together with the mean data from the last two prior tests obtained with the 36dot matrices. The transfer gradients obtained with the 64dot matrices are similar to those obtained with the 36-dot matrices. They are, in fact, somewhat steeper, which may reflect the additional discrimination training that preceded each of the 64-dot tests.

There was no consistent effect of the changes in the stimulus patterns on the total response output during the test sessions. For 1 pigeon this value declined by about two thirds on the first session of each transfer test. For a 2nd bird, there was little change during the first transfer test, but a marked decline during the second. The 3rd pigeon, which received only one transfer test, showed a remarkable increase in test responding. It doubled its usual level of responding during gradient tests. None of these changes appear to have had a systematic effect on the form of the transfer gradients when compared with the prior relative gradients obtained with 36 elements.

\section{Discussion (Experiments 1 and 1A)}

Experiments 1 and $1 \mathrm{~A}$ showed that if pigeons are trained to discriminate between spatial uniform arrays of distinct elements, the relative proportions of these elements will control responding in an orderly fashion. The transfer observed between these two experiments strongly suggests that the pigeons were controlled by the relative rather than the absolute numbers of dots of each kind. If they had been controlled by the absolute number of positive dots, the gradients would have been much flatter and shifted to the right during the transfer test, as there were, of course, many more positive dots in the 64-dot arrays than

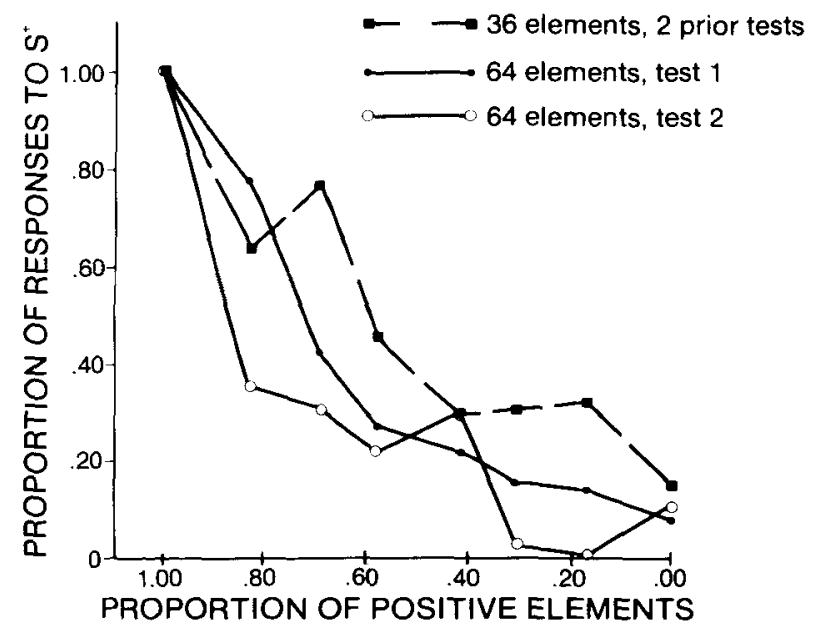

Figure 3. The mean relative numerosity gradients from the transfer test with 64 small dots in Experiment 1A. The mean of the two preceding tests with the 36-dot training arrays is shown for comparison. in the 36-dot matraices. Conversely, if they had been controlled by the number of negative dots, the transfer gradients would have been very steep and shifted to the left. There was, in fact, a modest shift toward the "positive end," but this most likely reflects a general enhancement of stimulus control due to repeated testing, which is also seen with the successive tests with 36 dots. Experiment 3 provided a similar effect of repeated testing with a different stimulus dimension.

These findings, then, instantiate a discrimination based on the relative numbers of different stimulus elements. This requires a discrimination of the classes of elements, and some kind of process of enumeration of each class to permit an assessment of their relative numbers. Subsequent experiments addressed some of the possible mechanisms of enumeration as we extended the present paradigm to include elements that differ on other stimulus dimensions.

\section{EXPERIMENT 2: FORM DISCRIMINATION}

Experiment 1 involved simple stimuli differing in color. In additional experiments, we used other stimulus dimensions for numerosity discriminations to extend the generality of the phenomenon, and perhaps to provide some clues regarding the mechanism by which numerosity discriminations with small elements are accomplished. The pigeon may have summed the areas of the individual dots of each color. Then it could make a numerosity discrimination by comparing the two areas. This would not trivialize our results; however, it would suggest that number discrimination is not a "primary" process of stimulus analysis for the pigeon, but is derived from a different and presumably more fundamental perceptual mechanism.

We addressed this possibility by using, in Experiment 2, stimuli that differed in form. We used different typed letters: $X$ and $O$. It is not apparent how the pigeon could sum the instances of these elements to encode them in a different form. The general method was the same as that used in Experiment 1: The pigeons discriminated two uniform arrays composed of the different elements, and were then tested with mixtures in different proportions. They were then given a transfer test with arrays composed of a smaller number of elements.

\section{Method}

Four new pigeons were used for this research. One failed to acquire the basic discrimination and was dropped from the study. The displays used for training and the first test comprised the capital letters $X$ and $O$ arranged in matrices of 36 elements. The letters were $4 \mathrm{~mm}$ wide and $6 \mathrm{~mm}$ high on the response panel. One set of these arrays is shown in Figure 1. The arrays were $52 \mathrm{~mm}$ wide and $55 \mathrm{~mm}$ high. The elements occurred in the same proportions as the colored dots in Experiment 1. The test series included arrays of the two forms in equal proportions (18 of each). We prepared four sets of arrays by typing them on paper, making transparent photocopies, and mounting them as slides for the projector. For transfer tests, matrices of $16 \mathrm{Xs}$ and $\mathrm{Os}$ were prepared with a larger style of type. The letters were $6 \mathrm{~mm}$ wide and $10 \mathrm{~mm}$ high. 
The transfer arrays were $51 \mathrm{~mm}$ wide and $50 \mathrm{~mm}$ high. For the transfer testing, a linear sequence of proportions of elements was used: $16 \mathrm{Xs}, 0 \mathrm{Os} ; 2 \mathrm{Xs}, 14 \mathrm{Os} ; 4 \mathrm{Xs}, 12 \mathrm{Os} ; 6 \mathrm{Xs}, 10 \mathrm{Os} ; 8 \mathrm{Xs}$, 8 Os; $10 \mathrm{Xs}, 6$ Os; $12 \mathrm{Xs}, 4 \mathrm{Os} ; 14 \mathrm{Xs}, 2 \mathrm{Os} ; 16 \mathrm{Xs}, 0$ Os.

The general procedures were the same as those used in Experiment 1. After they had acquired keypecking, the pigeons were trained to discriminate between uniform arrays of Xs and Os. For 2 of them, the Os were positive; for the 3rd, Xs were positive. Each bird received 12 training sessions. Each session comprised 32 trials, 16 with each display, presented in a randomized order.

Two consecutive test sessions were then carried out in extinction with the 36-element displays. Each comprised four randomized blocks of the nine different proportions of elements. After three further training sessions with the original stimuli, each pigeon was given a two-session transfer test with the 16-element displays in nine different proportions. This was also carried out in extinction, and consisted of four blocks of trials in each session.

\section{Results and Discussion}

Of the 3 birds that acquired the discrimination, 1 was very quick, reaching a discrimination ratio (DR) of .99 within 4 sessions. The others were slower, and reached a DR of .90 or more after 9 or 10 sessions. The data from the first test were summed across the two test sessions, and the average is shown as a normalized gradient in Figure 4. There is a single inversion, but otherwise the gradient is orderly and almost linear. The data from the 16-element transfer test are shown in the same figure. The gradients are generally similar, except that the transfer gradient is somewhat lower when the number of negative elements exceeded the number of positive elements. This could be an effect of repeated testing: when the data from the 2 test days with the 36-element arrays are plotted separately (not shown here), there is a similar reduction of responding at the negative end of the gradient during the second test session. Another gradient obtained with

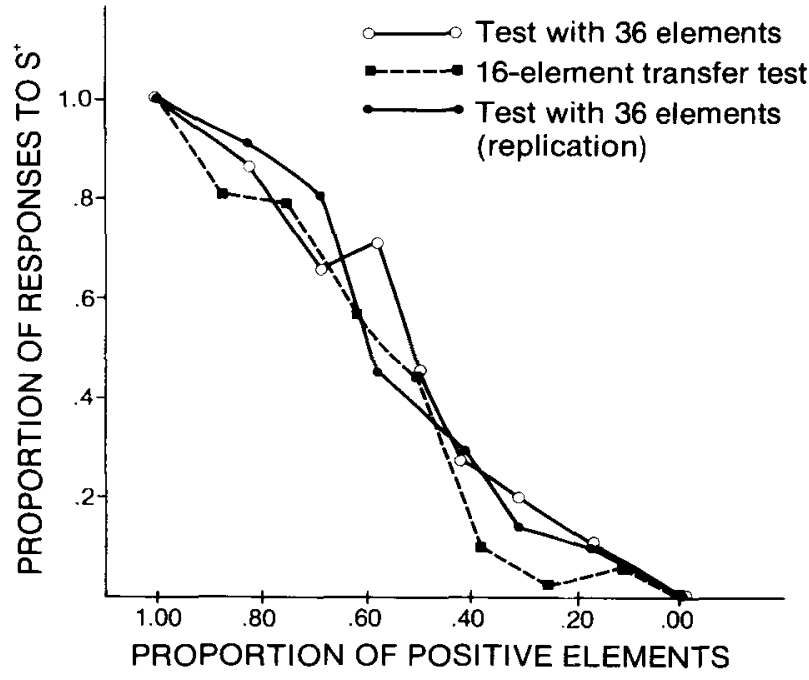

Figure 4. Mean relative numerosity gradients from 3 pigeons in Experiment 2 with different forms. Circles represent data from different groups of birds tested with 36 elements (see text). The dashed line shows data from a transfer test with arrays of 16 larger elements.
36 elements (Xs and Os) also is shown: This was obtained from 4 pigeons in a later experiment with the same method (but not otherwise described here), and is included for the sake of comparison. This replication provided a gradient very similar to that obtained in the first instance, and suggests that our results are reliable.

Two of the 3 birds responded more in the transfer test than in the training test: 1 increased the response output from 946 to 1,415 responses, the other from 2,248 to 2,822 responses. The 3 rd subject showed a huge reduction in response output, from 3,039 to 350 responses. Thus, at least 1 subject "noticed" the difference between the arrays in the form of a generalization decrement.

The data from the transfer test provide a systematic replication of the transfer tests obtained with the 64-dot arrays in Experiment $1 \mathrm{~A}$, in which an increase in the number of elements had little effect on gradients based on the proportions of positive elements. In Experiment 2, a considerable decrease (of about $55 \%$ ) in the number of elements again had little effect. These transfer data again suggest that the pigeons were responding on the basis of the relative rather than the absolute numbers of the elements. Control by the absolute number of positive or negative elements would have shifted the gradient to the right or the left, respectively, but no such shift was observed. The data that have been included from a subsequent experiment provide an impressive replication of the orderly control by the relative numbers of Xs and Os.

The present findings support the discrimination of numerosity as a primary, or direct, process. The pigeons appear to enumerate the different forms as individual elements. There is no simple way in which the subject could sum across the elements that are alike in form, in the way that summing across the areas of the colored elements may be possible. This does not, of course, imply that the process of enumeration is necessarily the same for colors and forms; similar data from different sets of stimuli could be obtained from different mechanisms of perceptual analysis.

\section{EXPERIMENT 3: SIZE DISCRIMINATION}

The discrimination of the numerosity of elements that differ in size would seem to be of adaptive importance. The relative numerosities of large and small items of a particular class, such as beach stones, may provide cues for geographical locations. The selection of patches for foraging may be affected by the numbers of large or small candidates for consumption. The size discrimination is also of theoretical importance in the present context. It provides a further and more stringent test of the hypothesis that numerosity discriminations are accomplished by the subject by summing across the items in each class, so that they are recoded in terms of their total area. If the pigeon sums across elements that differ in size, but that are identical in color and shape, a numerosity discrimination based on size would be impossible. 
In our experimental situation, a size discrimination involves some inherent confounds. The total area of a fixed number of elements increases with the size of the elements. The distances between the large items also are smaller. We were unable to compensate for this by presenting more small than large items in the training arrays, because the pigeons could then base the discrimination on the absolute numbers of items. The size discriminations were designed to obviate these confounds to some extent, and our data generally suggest that the subjects based their numerosity discriminations on the sizes of the elements, rather than on some other confounded feature of the display.

\section{Method}

Subjects. Six new pigeons were used for this experiment. They were maintained at $80 \%$ of their free-feeding weights. One of them failed to learn the discrimination between small and large dots, and was dropped from the experiment.

Stimuli. Arrays of red dots were used. The small dots were the same as those used in prior experiments. The diameter of the large dots was 2.37 times as great, and their area 5.64 times as great. In training, the small dots were presented in square arrays of 36 , 25,16 , and 9 . The large dots were presented in square arrays of $25,16,9$, and 4 . Each of these arrays was distributed over the entire display screen, except for the arrays of four large dots. The latter were concentrated in one quadrant, to keep the interelement distances comparable to those of the other arrays. This matrix was presented in different quadrants on various trials.

The uniform arrays of large and small elements overlapped considerably with respect to their number, namely 9,16 , and 25 . The overlap in total area among the arrays was not as great: The 4 large dots covered about the same area as 23 small ones, but even 9 large dots covered about the area of 51 small ones, more than the maximum of 36 presented in training.

Three sets of mixed arrays were used for testing, again with the aim of presenting patterns that differed in total area. These were matrices of 9,16 , or 25 elements. Six uniform training arrays were also used as test patterns: 9 large, 9 small, 16 large, 16 small, 25 large, and 25 small. The mixed test patterns were as follows: 3 large and 6 small, and 6 large and 3 small (for a total of 9); 4 large and 12 small, 8 large and 8 small, and 12 large and 4 small (for a total of 16); 20 large and 5 small, 15 large and 10 small, 10 large and 15 small, and 5 large and 20 small (for a total of 25). All of these patterns were made up with four different randomized arrangements of dots. A few sample uniform and mixed patterns are shown in the lower panel of Figure 1.

Procedure. Autoshaping was required for only 2 of the 5 pigeons. Each bird was trained for eight sessions with either large or small dots to acquire a stable rate of responding to its positive arrays. Three birds were trained with large dots positive, and 2 with small dots positive. Each session comprised 32 trials. The four positive arrays were presented eight times each, in a randomized order. Discrimination training followed this phase of acquisition. The four positive and four negative arrays were intermixed in four blocks of training trials; thus a subject received 4 trials each with 25,16 , 9 , and 4 large dots, and with $36,25,16$, and 9 small dots. After each bird reached a stable level of discrimination, it received the first test with all the combinations of 9 and 16 elements listed above. These were the four uniform arrays of 9 and 16 small and large elements, and five mixed arrays, two of them consisting of 9 elements and three consisting of 16 , as specified above. These patterns were presented in four blocks of randomized sequences of 9 trials each. This test was carried out without reinforcement for two sessions.
After eight further training sessions, each bird received a second test, this time with the arrays of 25 elements. The two uniform and four mixed arrays were each presented once in each block of trials. Two test sessions consisted of four blocks of trials. After five further training sessions, each subject received a third test that contained all of the arrays of 25 and 16 elements. This test involved the four uniform arrays and seven mixed arrays. Three of these contained 16 dots, and four contained 25 dots, in the proportions described above. The test was carried out for two sessions without reinforcement.

\section{Results}

Discrimination training. Of the 5 birds that learned the discrimination, 3 did so remarkably quickly. They each attained a DR greater than .90 by the 4th session. They were then run for a few more sessions, and their performance was essentially perfect. A 4th subject was run for 17 sessions before meeting the criterion of 3 successive sessions with a DR of .90 or greater. A 5th subject reached a DR over . 80 after 9 sessions, but then improved slowly. It met the criterion after 33 sessions, and was run only on the second and third gradient tests described above. The subjects maintained the trained discrimination very well during the training sessions that intervened between the three tests.

Numerosity gradients. Figure 5 presents the five averaged relative numerosity gradients at the left. These were obtained from the 9- and 16-element arrays in Test 1, from 25-element arrays in Test 2, and from 16- and 25element arrays in Test 3 . In all cases, orderly gradients were obtained. They became steeper with extended training and repeated testing, as is evident in a comparison of the two gradients obtained with 16 and with 25 elements at different times. There is no doubt that pigeons can readily discriminate the relative numerosities of elements that differ in size, and this dimension serves as a basis for orderly stimulus control.

Two comparisons address the question of whether the pigeons' discrimination of numerosity was independent of the total area of the elements in the array. One is based on training data with the uniform arrays. If the discriminations are based in part on area, and if large dots are positive, the birds should respond more to arrays containing more of them (25) than to arrays containing fewer (4), since the smaller number involves a smaller area. The opposite should be the case if small dots are positive. We examined the response totals from the three training sessions that preceded each of the gradient tests. The data are shown in Table 1. They are arranged so that the numbers should decrease from left to right if total area is a factor in determining response rates. There is no suggestion of any such trend.

Another comparison is based on test results. In two of the tests, matrices with different numbers of elements were presented concurrently: 9 and 16 in the first test and 16 and 25 in the third. If the pigeons were controlled, at least in part, by the total areas of the elements in the array, then the birds trained with large dots as positive ought to respond less, for a given proportion of elements, 


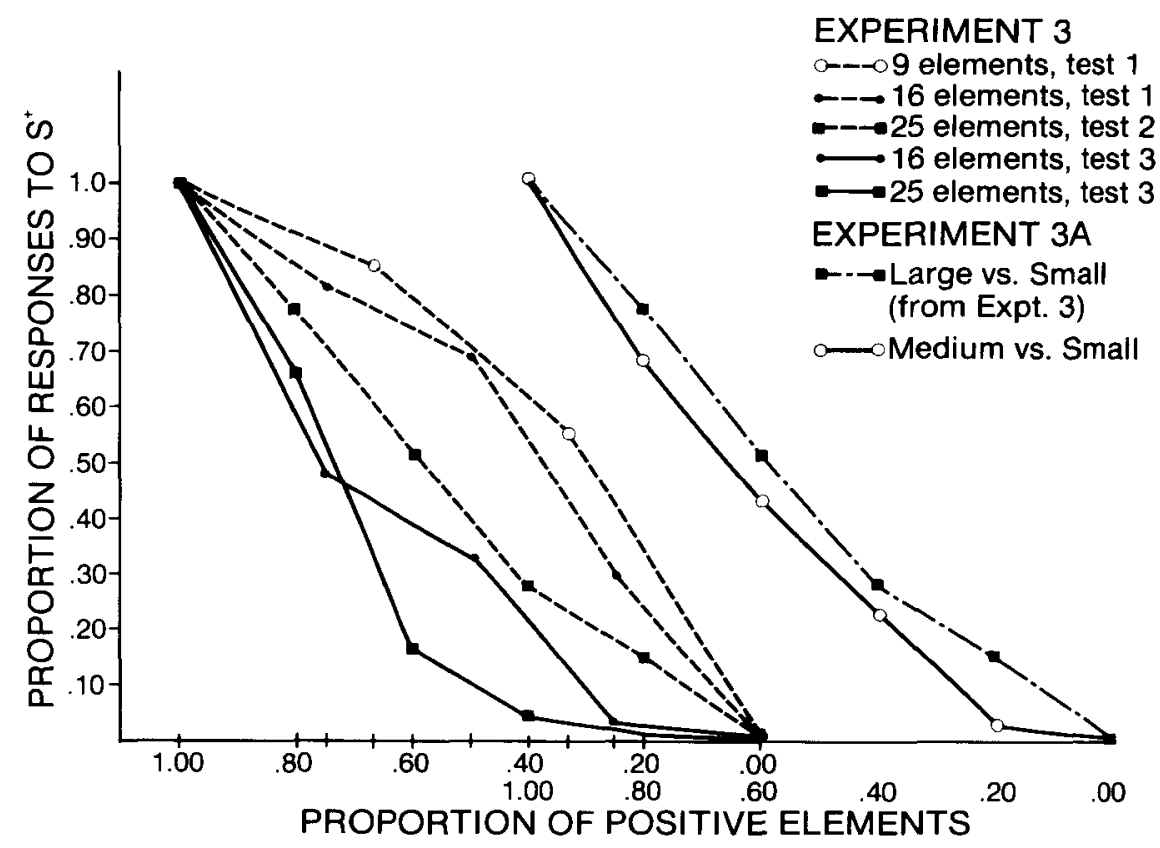

\begin{abstract}
Figure 5. At the left are the mean relative numerosity gradients obtained from three tests with arrays of small and large dots in Experiment 3. Arrays of 9, 16, and 25 dots were presented in different tests. At the right is the gradient obtained with 25 medium and small dots in Experiment 3A. The gradient from the second test (with 25 dots) in Experiment 3 is plotted again to provide a comparison.
\end{abstract}

to the less numerous than to the more numerous arrays in a gradient test. The opposite would be the case for birds trained with small dots as positive. The appropriate comparisons are presented in terms of mean total responses in Figure 6. The birds trained with large dots positive did not respond less to the 9-element arrays than to the 16element arrays in the first test. The data from the third test, with patterns of 25 and 16 dots, were not quite as orderly, but again there is no systematic difference between total responses to the two kinds of patterns. The 2 birds trained with small dots positive did respond slightly more to the 9-element arrays in the first test, but the difference is not convincing. In the third test, they responded reasonably well with the 16-dot arrays, but very little to the 25-dot arrays. This is the one case in which the difference in responsiveness was correlated with the areas of the arrays comprising different numbers of elements.

\section{EXPERIMENT 3A: TRANSFER TO ARRAYS OF MEDIUM AND SMALL DOTS}

It also was of interest to determine whether numerosity gradients are affected by the discriminability of the elements in the arrays. A systematic study of this question would involve more than one dimension, and probably an extended series of experiments. The present experiment was an initial attempt to address this question. We took advantage of the fact that differences in size can readily be scaled, unlike differences in color or form. The large dots used in the prior experiment were replaced by dots of medium size, $6 \mathrm{~mm}$ in diameter on the response screen. (The large dots were $9 \mathrm{~mm}$ in diameter.) The small dots were unchanged. Matrices of 25 dots were used, with test mixtures of the same proportions as in Experiment 3. After a layoff of some months, the same birds were trained with the arrays of medium and small dots. For the birds with small dots positive in Experiment 3, the small dots were still positive. For the birds with the large dots positive in Experiment 3, the medium dots were positive in the present study. Seven training sessions were carried out. The pigeons were tested on 2 consecutive days with the uniform and mixed arrays of 25 elements.

\section{Results}

The pigeons rapidly acquired the discrimination between the medium and small dots. They achieved DRs of .95 or above by the end of the seven training sessions. The single gradient obtained from the generalization test is shown in the right part of Figure 5, together with the gradient from the second test (with 25 elements) in Experiment 3 . The test procedure differed only in the sizes of the larger elements. The mean gradients are very similar. However, the gradient obtained from the second test is somewhat steeper. This is most likely due to repeated testing, and not to the characteristics of the stimuli. The control of responding by the relative numbers of elements differing in size seems to be unaffected by the magnitude of this difference. If the dots were made very similar in size, the gradient might take a different form, but the present study did not reach the limits of the pigeons' capacity for discriminating on the dimension of size. 


\section{Discussion (Experiments 3 and 3A)}

The stimulus patterns and the training and test procedures used in Experiments 3 and $3 \mathrm{~A}$ differed somewhat from the work that established numerosity gradients based on color and form. Because of the constraints inherent in presenting stimuli that differ in size, smaller numbers of items were presented in the training and test matrices. Furthermore, that number varied in training in a manner orthogonal to the dimension of interest, namely the size of individual items. In spite of these constraints, orderly data based on the relative numerosity of large and small elements were obtained from the individual subjects. The relative gradients were affected more by repeated testing than by the numbers of items in the test arrays. Our analysis of the data indicates that for the most part, the pigeons were under the control of numerosity of the items rather than the total area. This is actually a rather striking finding, since the training procedures involved equal reinforcement to several training arrays that differed in number of elements. Numerosity was therefore an irrelevant dimension in training. Nonetheless, we obtained excellent stimulus control on that dimension in testing.

In these experiments we trained and tested pigeons with different total numbers of elements. This provided a useful systematic replication of the transfer tests in Experiments 1 and 2, although that was not the main reason for

Table 1

Response Totals to Different Training Arrays Preceding Each Numerosity Test in Experiment 3 Large Dots Positive

\begin{tabular}{|c|c|c|c|c|c|}
\hline \multirow[b]{2}{*}{ Subject } & \multirow[b]{2}{*}{ Test } & \multicolumn{4}{|c|}{$\begin{array}{c}\text { Large Dots Positive } \\
\text { Array }\end{array}$} \\
\hline & & $25 \mathrm{~L}$ & $16 \mathrm{~L}$ & $9 \mathrm{~L}$ & $4 \mathrm{~L}$ \\
\hline \multirow[t]{2}{*}{4947} & $\begin{array}{l}1 \\
2 \\
3\end{array}$ & $\begin{array}{l}190 \\
186 \\
240\end{array}$ & $\begin{array}{l}221 \\
179 \\
220\end{array}$ & $\begin{array}{l}191 \\
214 \\
271\end{array}$ & $\begin{array}{l}304 \\
259 \\
293\end{array}$ \\
\hline & $M$ & 205 & 207 & 227 & 285 \\
\hline \multirow[t]{2}{*}{4568} & $\begin{array}{l}1 \\
2 \\
3\end{array}$ & $\begin{array}{l}200 \\
245 \\
260\end{array}$ & $\begin{array}{l}211 \\
244 \\
252\end{array}$ & $\begin{array}{l}190 \\
233 \\
242\end{array}$ & $\begin{array}{l}192 \\
254 \\
248\end{array}$ \\
\hline & $M$ & 235 & 236 & 222 & 231 \\
\hline \multirow[t]{2}{*}{4181} & $\begin{array}{l}1 \\
2\end{array}$ & $\begin{array}{l}142 \\
115\end{array}$ & $\begin{array}{l}151 \\
151\end{array}$ & $\begin{array}{l}172 \\
138\end{array}$ & $\begin{array}{l}187 \\
151\end{array}$ \\
\hline & $M$ & 128 & 151 & 155 & 169 \\
\hline \multirow[t]{3}{*}{$3 \mathrm{Ss}$} & $M$ & 189 & 198 & 201 & 228 \\
\hline & & \multicolumn{4}{|c|}{$\begin{array}{l}\text { Small Dots Positive } \\
\text { Array }\end{array}$} \\
\hline & & $9 \mathrm{~S}$ & $16 S$ & $25 S$ & $36 S$ \\
\hline \multirow[t]{2}{*}{232} & $\begin{array}{l}1 \\
2 \\
3\end{array}$ & $\begin{array}{r}205 \\
156 \\
81\end{array}$ & $\begin{array}{l}179 \\
173 \\
102\end{array}$ & $\begin{array}{r}199 \\
180 \\
77\end{array}$ & $\begin{array}{r}134 \\
163 \\
91\end{array}$ \\
\hline & $M$ & 147 & 151 & 152 & 129 \\
\hline \multirow[t]{2}{*}{26} & $\begin{array}{l}1 \\
2 \\
3\end{array}$ & $\begin{array}{r}87 \\
99 \\
137\end{array}$ & $\begin{array}{r}82 \\
93 \\
123\end{array}$ & $\begin{array}{l}121 \\
127 \\
114\end{array}$ & $\begin{array}{r}94 \\
102 \\
109\end{array}$ \\
\hline & $M$ & 108 & 99 & 121 & 102 \\
\hline $2 \mathrm{ss}$ & $M$ & 125 & 125 & 136 & 115 \\
\hline
\end{tabular}

Note $-\mathrm{L}=$ number of large dots in the array. $\mathrm{S}=$ number of small dots in array.

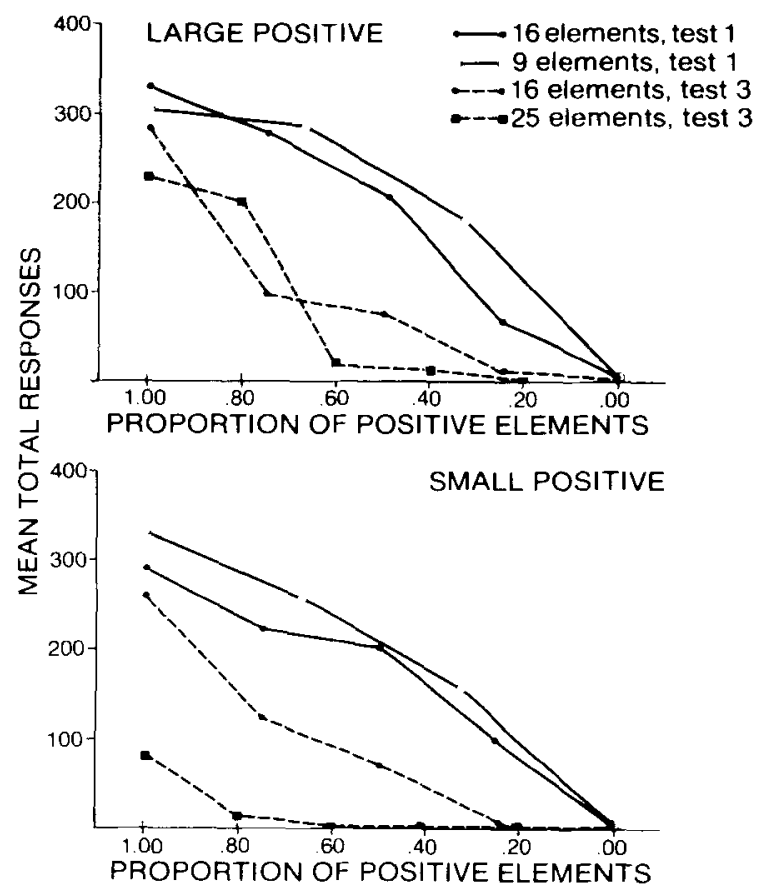

Figure 6. Mean total responses to the matrices of 9 and 16 elements in the first test of Experiment 3, and to the matrices of 16 and 25 elements in the third test. The data from the birds with large dots positive are shown in the upper panel, and from the birds with small dots positive in the lower panel.

presenting arrays of different total numbers. In those experiments, either more elements (64 colored dots) or fewer elements ( 16 forms) were presented instead of the 36 used in training. The results from the present experiment are similar and quite clear: the pigeons were controlled by the relative rather than the absolute numbers of the elements.

These considerations should not obscure the fact that the size discrimination was undertaken as a stringent test of the hypothesis that pigeons may "encode" the number of stimuli of each category in terms of total area. Such a mechanism would make it impossible for the animal to make numerosity discriminations based on size, when color and form are the same for both kinds of elements. Clearly, our subjects readily mastered the size discrimination, and provided orderly gradients of stimulus control. Numerosity judgments are not readily accounted for by some alternative, simpler mechanism.

\section{EXPERIMENT 4: CATEGORICAL DISCRIMINATIONS}

Simple, "elementary" stimuli were presented in the first three experiments. These are readily described, and different values from the same dimension-color, form, or size-were presented, so that discriminability between the stimuli could serve as an independent variable for numerosity discriminations. (We took advantage of this feature with different sizes of elements in Experiments 3 
and 3A.) Such discriminations may be facilitated if the elements from a given class are all alike. However, the real world does not come in tidy categories of uniform stimuli, and the ability to discriminate numerosities may be most useful with items from different "natural" categories. For example, a bird may identify a particular location from the relative numbers of deciduous and coniferous trees, which obviously differ among themselves. The same goes for human beings on a beach, where the relative numerosities of the two genders can determine where a new arrival will settle down. In the present study, we approximated such situations by using pictures of two kinds of natural stimuli: birds and flowers. They were arranged in matrices of 16 elements, and we used the same general procedures that provided the discrimination gradients in Experiments 1 and 2.

\section{Method}

Subjects and Stimuli. Four new pigeons were used for this experiment. They had had no experience with pictorial stimuli. The stimulus arrays were prepared from small round stickers, available as school supplies. These come in various natural categories, such as puppies, flowers, and unicorns. For the present experiment, we used colored sketches of individual flowers and birds. Sixteen different examples were chosen from each category. They differed in color and shape among themselves. Four different arrangements from each category were prepared from the stickers for training, photographed in color as slides, and projected onto the response panel. On the whole, the drawings of birds were more "solid" than those of flowers, and the colors somewhat bolder and more satu- rated. A sample array is shown in Figure 7 in a black-and-white copy. This is a mixed matrix of 8 birds and 8 flowers, and represents half of the instances of each category. The elements were $9 \mathrm{~mm}$ in diameter on the response panel, spaced $13 \mathrm{~mm}$ apart, center-tocenter.

The uniform arrays of 16 birds and 16 flowers served both as training stimuli and as test patterns. The other test patterns were prepared in the same proportions as the arrays of 16 elements in Experiment 3: 16 birds; 12 birds, 4 flowers; 8 of each kind; 4 birds, 12 flowers; 16 flowers. Figure 7 illustrates one of these. Specific elements were randomly selected for each test pattern and randomly located in it. However, each of the 16 different instances (or elements) from each category was used in the construction of each set of test slides before any was repeated. Thus, the specific instances on any particular test (or training) slide were all different.

Procedure. The birds were taught in regular training to peck at a slide containing the positive category. They were then trained on the standard extended but discrete trials for five sessions of 32 trials each, in which only positive arrays were presented. Each session comprised eight blocks of the four training patterns. After five sessions, the birds received regular discrimination training with the all-bird and all-flower slides. There were four negative and four positive trials in each block of eight trials, and a session comprised four blocks.

When the birds reached the criterion on the discrimination, they were tested for two sessions without reinforcement. Each block of trials contained one example of each proportion of instances of the training elements. Four blocks of trials were run in each session.

\section{Results and Discussion}

The birds pecked readily at the complex stimulus patterns, and learned the categorical discrimination remark-

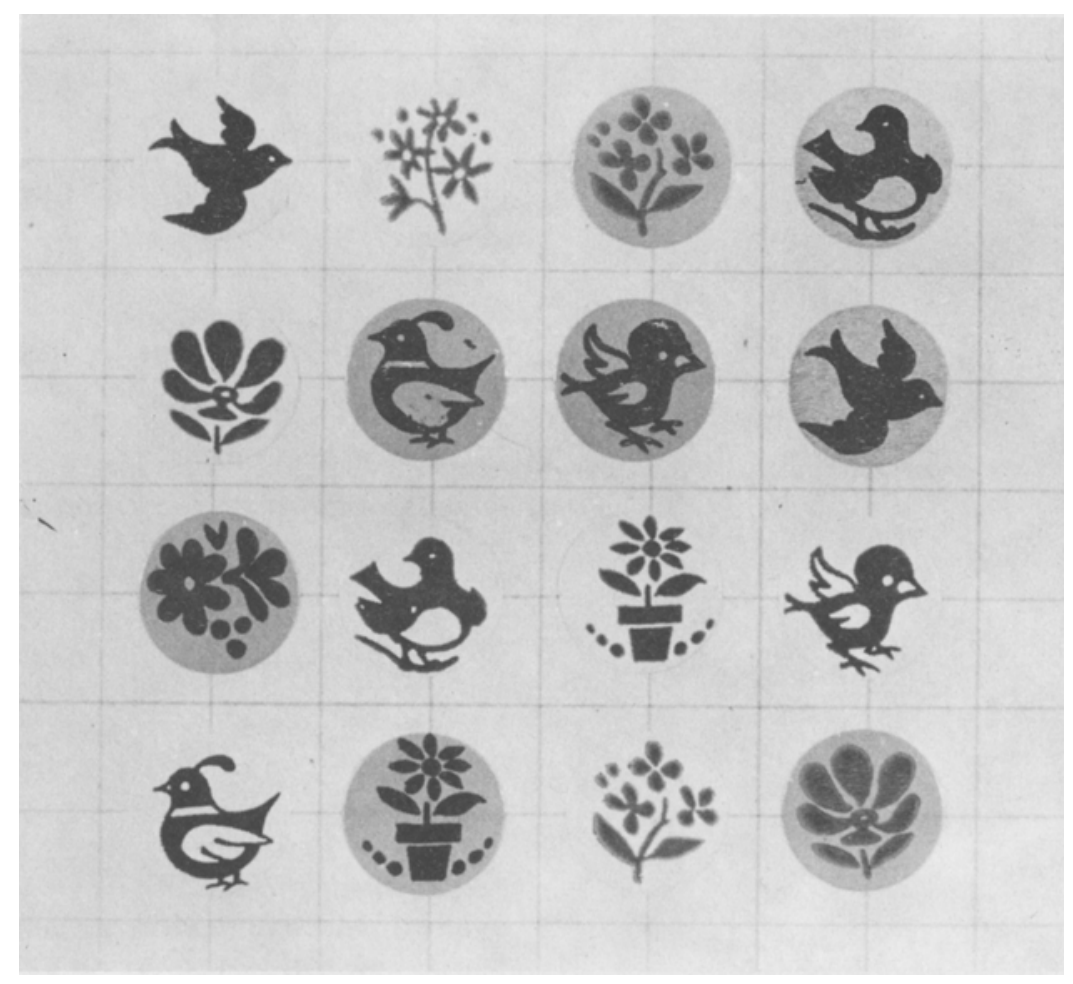

Figure 7. A sample test matrix of 8 flowers and 8 birds used in color for Experiment 4. Sixteen instances of each class were used in this study, and only half of them are shown here. 
ably quickly. No subject took more than three sessions before it acquired a DR of .90 or better. Three birds were trained for five sessions; 1 required six.

The discrimination gradients are shown for the 4 individual birds in Figure 8, together with the mean gradient. Three birds produced an orderly numerosity gradient. One had a marked bias toward the positive end of the gradient.

These results suggest that pigeons can assess the relative numerosities of complex stimuli that represent natural categories. We do not know, of course, whether our subjects identified the birds and flowers as natural categories; quite clearly, the little pictures differed in many ways from the objects that they represent, and the birds may have "constructed" the categories. But we do know that pigeons are adept at identifying stimuli from particular categories from pictures: Aside from the studies with human beings as the conceptual category (Edwards \& Honig, 1987; Herrnstein \& Loveland, 1964; Siegel \& Honig, 1970), trees (Vaughan \& Herrnstein, 1987), bodies of water (Herrnstein et al., 1976), fish (Herrnstein \& deVilliers, 1980), and man-made constructions (Lubow, 1974) have been used. In all of these experiments, pictures were presented. Thus, although the sketches used for our work were not photographs, they may still have been identified by the subjects as birds and flowers.

The pigeons could have learned the discrimination by attending to one or a few of the specific instances of each category, since in training all birds appeared in each bird slide, and all flowers appeared in each flower slide. Then, in testing, the bird would have found a particular positive instance on three fourths of the slides containing 12 positive instances, on half of the slides containing 8 positive instances, and on one fourth of the slides containing 4 positive instances. Thus, the gradients could be a statistical artifact reflecting specific stimulus control by a small

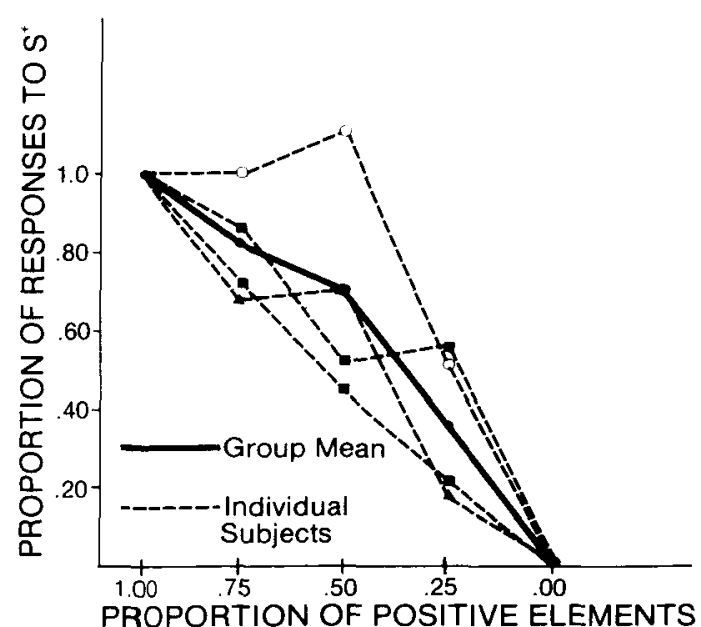

Figure 8. Mean relative numerosity gradients obtained with different proportions of birds and flowers as stimuli in the discrimination test of Experiment 4. The data from individual subjects are shown together with the group mean. number of instances. A trial-by-trial analysis of the data is required to examine this possibility, and this is not available. A stronger test is the transfer of the categorical numerosity discrimination to new instances, and this was done in Experiment 5.

\section{EXPERIMENT 5: TRANSFER BETWEEN INSTANCES OF A CATEGORICAL DISCRIMINATION}

\section{Method}

Four new pigeons were used for this research. Their prior experience did not involve any discriminations involving numerosities or categorical elements. New arrays of two conceptual categories were prepared with the kinds of stickers used in Experiment 4. Two independent sets ( $A$ and $B$ ) of elements were used to test for transfer with new instances. As the numbers of different instances were limited, each array comprised 9 rather than 16 elements. The categories were flowers and unicorns. (Unicorns replaced birds, because 18 different kinds of unicorns were available, and only 12 kinds of birds.) The arrays contained either 9 different flowers, 6 flowers and 3 unicorns, 3 birds and 6 unicorns, or 9 unicorns. The general procedures were similar to those carried out in prior experiments.

Bird 4980 was trained with unicorns from Set A positive, Bird 4944 with flowers from Set A positive, Bird 4817 with unicorns from Set B positive, and Bird 4802 with flowers from Set B positive. Each bird was first trained to peck at several different configurations of the positive training instances. One subject was run for seven sessions of acquisition; the other 3 for nine sessions. Discrimination training was then carried out in the usual manner. All subjects reached a high level of discrimination (DRs over .90) within nine sessions or less; unfortunately, 2 of them were misrun for two sessions after that, and it took them many sessions to re-attain their prior level of discrimination.

Two sessions of gradient testing were carried out in extinction. Eight different kinds of arrays were presented: There were two training and two transfer slides of uniform arrays of nine flowers or unicorns, and four mixtures of three of one kind and six of the other, two of them composed of training elements and two composed of transfer elements. Four blocks of these arrays were presented in randomized order during each test session.

\section{Results and Discussion}

Since the tests involved both training and transfer stimuli, the gradients are shown in Figure 9, both in the usual normalized form and in terms of total test responses. Clearly, the discrimination transferred very well between the training and transfer arrays. There is a single discrepancy in the relative gradients at the test proportion of six positive and three negative elements. This is due to 1 bird (4817) that responded excessively to the arrays of six positive stimuli. Without this subject, the mean gradients would be all but identical. The gradients based on total responses are also quite similar. The mean total test responses (summing across all stimuli) were 726 for the training elements and 699 for the transfer elements. Although responsiveness varied widely among subjects, the general level of responding to the training and test stimuli was similar for each individual bird. There are also sizable differences among subjects in some of the proportional test values (see, e.g., the test data obtained with 


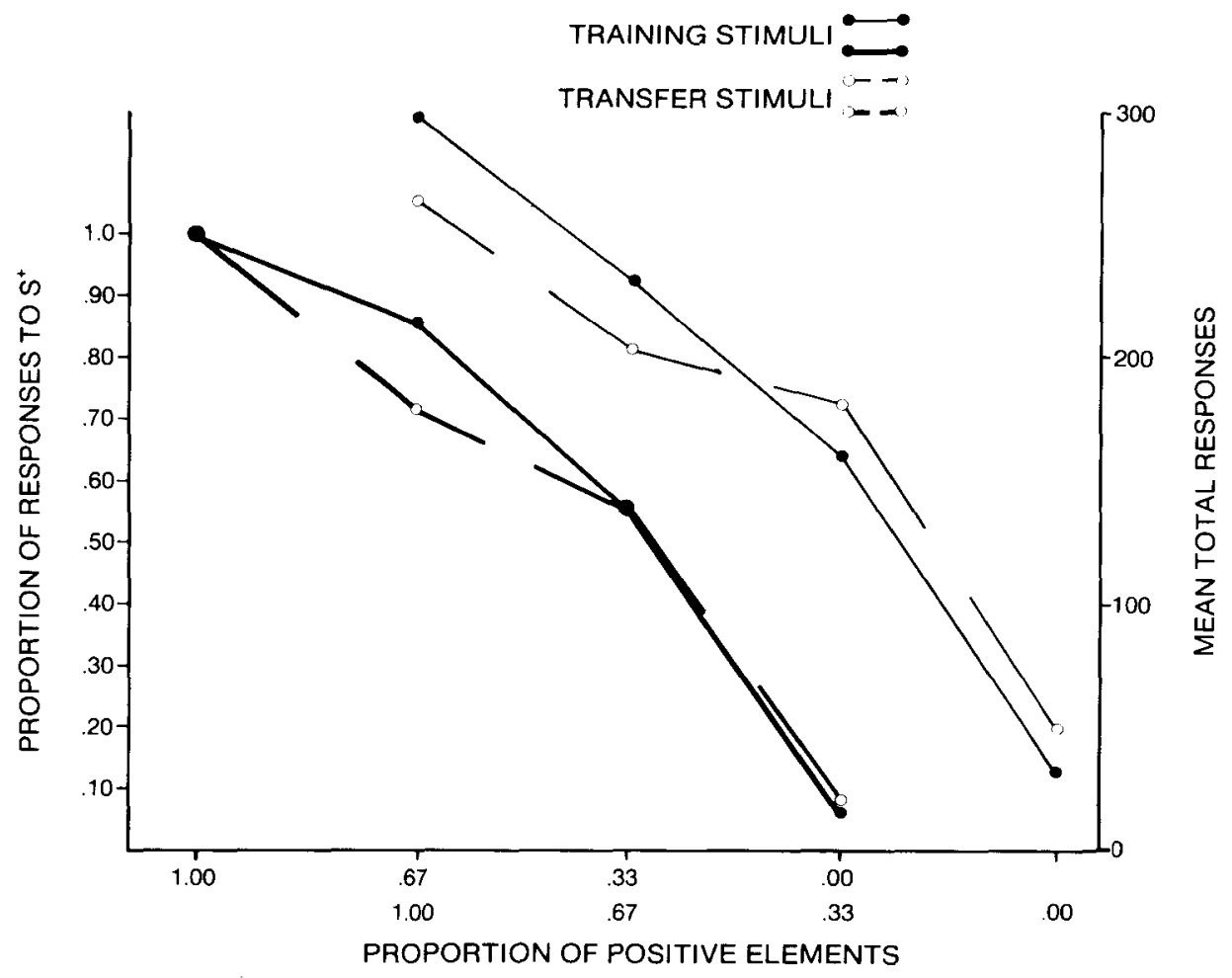

Figure 9. Mean numerosity gradients obtained with different proportions of flowers and unicorns in the discrimination test of Experiment 5 . The relative gradients are at the left; the gradients based on total responses are at the right.

arrays of three positive and six negative elements). However, we feel that this variability is a matter of sampling error in a rather complex gradient test, and that the birds recognized the similarity between the training and transfer elements, and discriminated them accordingly.

These transfer data are important, since they indicate that, in Experiment 4, the pigeons were not memorizing individual instances of the categories and then searching for the positive ones in the different test arrays. The total numbers of responses to the training and transfer stimuli were similar; there was no generalization decrement with the latter. The data from Experiment 5 therefore suggest that the discriminations were categorical, and that the relative numerosities of novel stimuli can readily be assessed, provided that such stimuli are identified as members of the appropriate categories.

\section{GENERAL DISCUSSION}

The present data represent systematic replications that establish relative numerosity as a dimension of stimulus control in pigeons. This form of stimulus control has been obtained with three primary stimulus dimensions, namely color, form, and size. Although the elements were chosen to differ on the basis of the nominal dimensional cues, other, confounded cues may be more salient for the pigeon than for the human observer. For example, dots of different colors differ also in brightness and perhaps saturation, and large elements have a longer circumference than small ones, as well as a greater area. The questions that remain about the salient features of the primary stimuli do not render invalid the most important finding: that numerosity, although it is a "derived" dimension based on an aggregation of individual stimuli, can govern behavior in much the same way as the dimensions on which these stimuli are individually distinguished. Experiments 4 and 5 demonstrated that numerosity discriminations are not limited to simple elements. These studies involved more complex pictures that resemble natural objects, and differ among themselves on several dimensions.

To provide favorable conditions for studying stimulus control with a new procedure, we carried out explicit discrimination training between the arrays representing the extreme values on the numerosity dimension. As a result, the gradients obtained with different primary dimensions were rather similar. The training procedures probably obscured differences in discriminability between different colors, forms, and sizes. In this respect, our work differed from many earlier experiments on stimulus control. Guttman and Kalish (1956) did not require differential training with their simple, monochromatic stimuli to obtain orderly generalization gradients, although the birds still had to discriminate the response key from its background. To enhance control on the dimensions of tonal frequency (Jenkins \& Harrison, 1960) and line orientation (Honig et al., 1961), the experimenters used interdimensional training, in which the presence of the positive stimulus was contrasted with its absence. We do not know what 
sort of numerosity gradients would be obtained with a less explicit training procedure-if, for example, the pigeon had to discriminate an array of red dots from an array of Xs in training, followed by a test with different proportions of red and blue dots. Procedures of this kind may reveal differences in the degree of stimulus control when various primary dimensions serve as "carriers" for the numerosity discriminations.

Other current research in our laboratory with a different training paradigm has in fact revealed marked differences in the control attained by elements from different dimensions. This is a "mixture" discrimination, in which the two uniform stimulus arrays are both positive and all mixed arrays are negative. In this procedure, neither kind of element in the array is associated differentially with reinforcement; the relationship between them determines this contingency. The pigeons learned this problem readily with red and blue dots, but found it much harder when Xs and Os or dots of different sizes were used. Special training procedures were required to enhance the mixture discrimination with these kinds of elements. Thus, there appears to be an interaction between the discriminability of the elements on different dimensions and the kind of training problem in which different relative numerosities are presented. The present experiments necessarily involved differential reinforcement and extinction of responding to the elements, and this may have enhanced the discriminability of elements differing in form or size.

Much of our research concerned the question of whether the process through which the numerosity discrimination was "accomplished" involved a summation of the areas covered by the different kinds of elements. Results of Experiments $2,3,4$, and 5 suggested that such a mechanism is not required. However, this does not mean that the pigeon would be insensitive to the sizes of elements in a numerosity discrimination that is based on some dimension orthogonal to size. "Bigger" may also appear to be "more." It would be of interest to present "oversized" elements in a numerosity discrimination based on color, keeping the relative numbers constant. Test arrays could be prepared with equal numbers of red and blue elements, but with larger red ones than blue ones. Would the pigeon be affected by this change as if it were an increase in the number of red elements? This is an important question, because an effective capacity for discriminating the numerosities of similar objects in the natural world should be relatively insensitive to their visual extent; size constancy should prevent a confusion between the extent of the array and the number of objects.

Our research was designed with the assumption that the pigeon would view the entire stimulus array and be controlled by its general features, rather than a few local cues. Other research from our laboratory with the same apparatus supports this supposition. Edwards and Honig (1987) carried out a concept attainment study with naturalistic scenes in which the presence of a human being in the picture was the positive feature. One training condition involved "matched pairs" of slides. One slide contained the human being, and the other presented the same background without the human feature. The pigeons learned the discrimination much more slowly and much less well in the latter condition. Although they could have "concentrated" on the human feature, their discrimination was clearly influenced by all the other cues that were common to the positive and negative arrays.

Nonetheless, it is conceivable that the pigeon attended only to a restricted portion of the display. In particular, it may have autoshaped to the small, punctate, positive elements, as these were differentially associated with reinforcement. If so, it would have found such elements at which to peck in all arrays except the uniform negative array. This would have produced a rather flat discrimination gradient, dropping precipitously between the arrays that contained the fewest positive elements and the all-negative array. The outcome was of course very different; the pigeons were strongly affected by the different numbers of negative elements.

Another "punctate" interpretation of our results is this: The pigeon pecked consistently at a particular spot on the slide, viewing only a small area. On a statistical basis, the few dots appearing in this area would contain the same proportions of negative and positive elements as did the overall array. Although responding might vary widely among individual trials, the average would reflect the general characteristics of the various arrays. To disprove this hypothesis, one needs to record the location of the pigeon's pecks, in conjunction with a trial-by-trial account of responding to the particular arrays. We do not have these sophisticated measures available at this time. Since we have obtained stable data with a wide variety of numbers and "densities" of elements, ranging from 9 to 64 , we do not find this version of a punctate hypothesis at all convincing, but a direct refutation is not within our grasp.

Our exposition of this material has not involved the concept of counting. We described the process as a discrimination of numerosity to avoid the implication that any concept of number was involved. There has been much recent work by Church, Meck, and Gibbon (see the review by Church \& Meck, 1984) on the discrimination of numbers of sequential stimuli. These numbers are much smaller than most of those presented to the pigeons in our work. Church and Meck stressed the functional equivalence of counting and timing in their work. Indeed, the sequential presentation of stimuli usually involves a confound between number and duration, either of total stimulus-on time, total presentation time, or both.

The simultaneous presentation of stimulus elements, as in the present research, eliminated any explicit relationship between number and presentation time. However, it is possible that the pigeon may have "enumerated" the number of positive (or negative) elements through a sequential search of the arrays. This could be assessed indirectly by using a discrete-trial conditional matching procedure. The pigeon would assess an array as a sample, and then respond to one of two comparison keys. The 
latency of this choice may be related to various aspects of the array: the total number of elements, the number of positive or negative elements, or the proportion of elements of one or the other kind. Certainly, a development and refinement of the present, rather straightforward methodology will be necessary to provide an analysis of the process used by the pigeon to estimate the relative numerosity of items in an array.

In the present discriminations, the numbers of total elements in an array were fixed in order to study the discrimination of relative numerosity with as few inherent confounds as possible. Natural circumstances do not provide such a neat abstraction of the present variable of interest. In real life, different numbers of the members of a given class are often confounded with other variables, such as the extent of the array and the variety of instances. It will be of interest in further research to study the discrimination of absolute as well as relative numerosities in a set of items. It is not a foregone conclusion that animals will discriminate numerosity on the basis of the simpler variables that are often confounded with numerosity. They may have evolved the capacity to discriminate groups of items on the basis of their numerosity, independently of other global features of the arrays. The present work suggests that the numerosity of similar items is a salient feature of stimulus arrays, and its discrimination should be useful in complex and extended natural environments.

\section{REFERENCES}

Church, R. M., Meck, W. H. (1984). The numerical attribute of stimuli. In H. L. Roitblat, T. G. Bever, \& H. S. Terrace (Eds.), Animal cognition (pp. 445-464). Hillsdale, NJ: Erlbaum.
Davis, H., \& Memmott, J. (1982). Counting behavior in animals: A critical evaluation. Psychological Bulletin, 92, 547-571.

EdWARDS, C. A., HoNIG, W. K. (1987). Memorization and "feature selection" in the acquisition of natural concepts in pigeons. Learning \& Motivation, 18, 235-260.

GreENe, S. L. (1983). Feature memorization in pigeon concept formation. In M. L. Commons, R. J. Herrnstein, \& A. R. Wagner (Eds.), Quantitative analyses of behavior: Discrimination processes (Vol. 4, pp. 209-229). Cambridge, MA: Ballinger.

Guttman, N., \& Kalish, H. I. (1956). Discriminability and stimulus generalization. Journal of Experimental Psychology, 51, 79-88.

HERRNSTEIN, R. J. (1979). Acquisition, generalization, and reversal of a natural concept. Journal of Experimental Psychology: Animal Behavior Processes, 5, 116-129.

Herrnstein, R. J., \& DeVilliers, P. A. (1980). Fish as a natural category for people and pigeons. In G. H. Bower (Ed.), The psychology of learning and motivation (Vol. 14, pp. 59-95). New York: Academic Press.

Herrnstein, R. J., \& Loveland, D. H. (1964). Complex visual concept in the pigeon. Science, 146, 549-551.

Herrenstein, R. J., Loveland, D. H., \& Cable, C. (1976). Natural concepts in pigeons. Journal of Experimental Psychology: Animal Behavior Processes, 2, 285-311.

Honig, W. K., Boneau, C. A., Burstein, K. R., \& Pennypacker, H. S. (1961). Positive and negative generalization gradients obtained after equivalent training conditions. Jourmal of Comparative \& Physiological Psychology, 56, 111-116.

JENKINS, H. M., \& HARRISON, R. H. (1960). Effect of discrimination training on auditory generalization. Journal of Experimental Psychology, 59, 246-253.

LUBOW, R. E. (1974). Higher-order concept formation in pigeons. Journal of the Experimental Analysis of Behavior, 21, 475-483.

SiEgel, R. H., \& HonIG, W. K. (1970). Pigeon concept formation: Success and simultaneous acquisition. Journal of the Experimental Analysis of Behavior, 13, 385-390.

VAUGHAN, W., JR., \& HERRNSTEIN, R. J. (1987). Choosing among natural stimuli. Journal of the Experimental Analysis of Behavior, 47, 5-16.

(Manuscript received May 19, 1988; revision accepted for publication October 14, 1988.) 